\title{
Production of biocatalysts and bioactive compounds from Greek basidiomycete wild strains grown in different induction media ${ }^{\dagger}$
}

\author{
Anastasia Zerva ${ }^{1}$, Nikolaos Tsafantakis ${ }^{2}$ and Evangelos Topakas ${ }^{1, *}$ \\ 1 Industrial Biotechnology \& Biocatalysis Group, School of Chemical Engineering, National Technical \\ University of Athens, 9 Iroon Polytechniou Str., Zografou Campus, Athens 15780, Greece; \\ anazer@chemeng.ntua.gr \\ 2 Department of Pharmacognosy and Natural Products Chemistry, Faculty of Pharmacy, National and \\ Kapodistrian University of Athens, Athens, 15771, Greece; ntsafantakis@pharm.uoa.gr \\ * Correspondence: vtopakas@chemeng.ntua.gr; Tel.: +30-2107723264 \\ + Presented at the 1st International Electronic Conference on Microbiology, 2-30 November 2020; Available \\ online: https://ecm2020.sciforum.net/
}

Published: 2 November 2020

\begin{abstract}
White-rot basidiomycetes are the only microorganisms with the ability to produce both hydrolytic (cellulases and hemicellulases) and oxidative (ligninolytic) enzymes for degrading cellulose/hemicellulose and lignin. In addition, they produce secondary metabolites with applications in the cosmetics industry. In the present work, three wild strains of Basidiomycete fungi (Pleurotus citrinopileatus, Abortiporus biennis and Ganoderma lucidum) from Greek habitats were grown in agroindustrial residues (oil mill wastewater, and corn cob). The production of biotechnologically relevant enzymes and bioactive compounds was evaluated. All strains were found to be preferential lignin degraders, similarly to most white-rot fungi. Bioinformatic analyses were performed on the proteome of the strains P. citrinopileatus and A. biennis, focusing on CAZymes with biotechnological relevance, and the results were compared with the enzyme activities of culture supernatants. The antiaging proprieties of the fungal extracts were evaluated in relation to the different media. P. citrinopileatus showed the most interesting skin whitening activity while $A$. biennis the highest DPPH scavenging potential. For all strains, corn cobs was the most appropriate carbon source for maximizing the production of bioactive small molecules with anti-aging activity. Overall, all three strains showed promising antiaging properties, and strong production of oxidative enzymes for biomass conversion applications.
\end{abstract}

Keywords: mushrooms; basidiomycetes; novel enzymes; biocatalysts; natural bioactive compounds; secondary metabolites; laccases; LPMOs

\section{Introduction}

White-rot basidiomycetes are the only microorganisms able to produce hydrolytic (cellulases and hemicellulases) and oxidative (ligninolytic) enzymes for attacking both cellulose/hemicellulose and lignin. Especially ligninolytic enzymes can be applied in numerous fields of industry, e.g. chemical, fuel, food, agriculture, and cosmetics industries. Lignin is recalcitrant to microbial degradation, but certain white-rot fungi are able to degrade lignin, cellulose and hemicellulose concomitantly, while others attack lignin selectively by secreting enzymes, namely phenol oxidases (laccases) and heme peroxidases [1]. White-rot fungi also secrete cellulases, xylanases and other hemicellulases. These enzymes act on long-chain polysaccharides, resulting in their hydrolysis. The substrates used for fungal growth are usually agricultural or agro-industrial residues of low economic value and possibly with toxic effects (e.g. olive-mill wastes). Recent studies revealed that 
the composition of cultivation substrates can significantly affect mushroom properties [2] or their antioxidant activity. Unfortunately, a huge gap still exists in our knowledge regarding the effect of cultivation substrates on the production of bioactive metabolites, and/or ligninolytic enzymes by edible/medicinal basidiomycetes.

Secondary metabolites are low molecular weight compounds (MW <1500), with structural and functional complexity. In the cosmetic sector they can be found in several antiaging formulations due to their anti-oxidant and anti-tyrosinase properties. Most of the tyrosinase inhibitors derive from natural sources including various plants and microorganisms. Among them, kojic acid, azelaic acid and ellagic acid are the most representative [3]. Tyrosinase is the key enzyme of melanin biosynthesis in microorganisms, plants, and animals. It plays an important role in the hydroxylation of L-tyrosine to L-3,4-dihydroxyphenylalanine (L-DOPA) and in the oxidation of L-DOPA to dopaquinone. Tyrosinase inhibitors are mainly used for the treatment and prevention of hyperpigmentation in the epidermal layers of the human skin. Despite the increase of clinical and industrial demands for tyrosinase inhibitors, nowadays there are very few compounds certified for clinical use [4]. Thus, there is a strong need for new anti-tyrosinase agents.

In the present work, wild strains of edible/medicinal saprotrophic basidiomycetes isolated from diverse habitats of Greek territory were evaluated for the production of cellulolytic and ligninolytic enzymes, and bioactive molecules with anti-aging (antioxidant and skin whitening) activity.

\section{Methods}

\subsection{Microorganisms and culture procedures}

The Pleurotus citrinopileatus LGAM 28684, Abortiporus biennis LGAM 436 and Ganoderma resinaceum LGAM 334 strains used for this study, were obtained from the fungal culture collection of the Laboratory of General and Agricultural Microbiology (Agricultural University of Athens). The strains were maintained in Potato Dextrose Agar plates at $4{ }^{\circ} \mathrm{C}$. Agar plugs (6 mm diameter) were used as inocula for the liquid cultures. Olive oil mill wastewater (OMWW) was obtained from an olive oil mill with a three-phase decanter in Kalamata (Peloponnese, S.W. Greece) and maintained at $-20{ }^{\circ} \mathrm{C}$ [5]. Prior to use, $\mathrm{pH}$ was adjusted to 6 with $3 \mathrm{~N} \mathrm{NaOH}$, and the suspended solids were removed. OMWW was added to the cultures at a final concentration of $50 \%(\mathrm{v} / \mathrm{v})$ with $100 \mathrm{mM}$ phosphate buffer $\mathrm{pH}$ 6. Alternatively, corn cobs or Avicel were added at a final concentration of $30 \mathrm{~g} \mathrm{~L}^{-1}$ as carbon source. Corn steep liquor $\left(3 \mathrm{~g} \mathrm{~L}^{-1}\right)$ was added as nitrogen source. Liquid cultures were incubated at $26{ }^{\circ} \mathrm{C}, 100 \mathrm{rpm}$. Samples were taken at selected time intervals, centrifuged (3000 rpm, $10 \mathrm{~min}$ ), and the supernatant was used for analysis and determination of enzyme activities.

\subsection{Extraction of fungal cultures}

For the evaluation of the antioxidant and skin whitening activity, biomasses were separated from culture supernatants by filtration using whatman filter paper No 4 . Biomasses were first lyophilized and then subjected to a sequential ultrasound-assisted extraction using Ethyl Acetate (EtOAc) and $50 \%$ Methanol/Water $\left(\mathrm{MeOH} / \mathrm{H}_{2} \mathrm{O}\right) .0 .4 \mathrm{~g}$ of dried biomass was extracted at room temperature for $40 \mathrm{~min}$ using $10 \mathrm{~mL}$ of solvent per cycle. Each sample was extracted 3 times, supernatants were centrifuged (4000 rpm, $3 \mathrm{~min}$ ), combined and dried under reduced pressure. Supernatants were subjected to a Liquid-Liquid extraction with EtOAc. $20 \mathrm{~mL}$ of each supernatant was extracted 3 times with $20 \mathrm{~mL}$ of EtOAc and the upper phases were combined and dried under reduced pressure. The aqueous part was lyophilized. In total, each fungal culture offered 4 different extracts: 2 from the biomass (EtOAc and $50 \% \mathrm{MeOH} / \mathrm{H}_{2} \mathrm{O}$ ) and 2 from the supernatants (EtOAc-LL and $\mathrm{H}_{2} \mathrm{O}$ ). Dried extracts were immediately stored at $-20^{\circ} \mathrm{C}$ prior to analysis.

\subsection{Enzyme assays and analyses}

Laccase activity was determined as described in [6]. Cellulase and xylanase activities were measured as described in [7]. The determination of reducing sugars was performed with the DNS method [8]. For the detection of LPMO activity, $200 \mu \mathrm{L}$ of culture supernatant were added to 
phosphoric acid-swollen cellulose (PASC) $1 \%(\mathrm{w} / \mathrm{v})$ in $50 \mathrm{mM}$ acetate buffer $\mathrm{pH}$ 5.2. Cysteine $(1 \mathrm{mM})$ was added as electron donor. The mixture was incubated for $16 \mathrm{~h}$ in $45^{\circ} \mathrm{C}$ and $1000 \mathrm{rpm}$. The reaction was terminated by boiling, and the supernatant was analyzed for oxidized sugars with HPAEC-PAD [9]. Phenols concentration was determined as previously described [6], and it was expressed in ppm of gallic acid equivalents. Decolorization was calculated by measuring the absorbance at 525 [6].

\subsection{Bioinformatics analyses}

Automated CAZyme annotation was performed to the predicted protein sequences of $P$. citrinopileatus and A. biennis, with the dbCAN2 meta server, employing the HMMER, DIAMOND and HotPep tools [10].

\subsection{Free radical scavenging (DPPH) and tyrosinase inhibition assay}

The protocol based on [11] was used as follows: $10 \mu \mathrm{l}$ of extract in DMSO $\left(4 \mathrm{mg} \mathrm{mL}^{-1}\right)$ and $190 \mu \mathrm{l}$ of DPPH solution $(0.314 \mathrm{mM})$ were mixed and incubated for $30 \mathrm{~min}$ at $25^{\circ} \mathrm{C}$ in the dark. Absorbance was measured at $517 \mathrm{~nm}$. Gallic acid was used as positive control. The percentage of DPPH scavenging was calculated as follows: [(A-B)-(C-D)]/(A-B) x100, where A : Control (w/o sample), B : Blank (w/o sample, w/o DPPH), C : sample, D : Blank sample (w/o DPPH). Extracts were tested at $200 \mu \mathrm{g} \mathrm{mL}-1$ and $100 \mu \mathrm{g} \mathrm{mL}-1$ (final concentration).

The anti-tyrosinase activity of the extracts was evaluated as described in [12]. Extracts were dissolved in DMSO and diluted in phosphate buffer 1/15 M, pH 6.8. In 96-well plates, $80 \mu \mathrm{l}$ of phosphate buffer $1 / 15 \mathrm{M}, \mathrm{pH} 6.8,40 \mu \mathrm{l}$ of sample and $40 \mu \mathrm{l}$ mushroom tyrosinase $\left(92 \mathrm{U} \mathrm{mL}^{-1}\right)$ were mixed and incubated for 10 minutes at $25{ }^{\circ} \mathrm{C}$, before $40 \mu \mathrm{l}$ of $2.5 \mathrm{mM}$ L-DOPA were added. After incubation at $25^{\circ} \mathrm{C}$ for $5 \mathrm{~min}$, the absorbance at $475 \mathrm{~nm}$ was measured. Kojic acid was used as positive control. The inhibition of the tyrosinase activity was calculated as follows: (A-B)-(C-D)]/(A-B) x100, where A : Control (w/o sample), B : Blank (w/o sample, w/o tyrosinase), C : Sample, D : Blank sample (w/o tyrosinase). Extracts were tested at $300 \mu \mathrm{g} \mathrm{mL}-1$ and $100 \mu \mathrm{g} \mathrm{mL}-1$ (final concentration).

\section{Results and Discussion}

The fungal cultures of A. biennis, P. citrinopileatus and G. resinaceum in different lignocellulosic waste materials as carbon sources were evaluated for their anti-oxidant and anti-tyrosinase acitivities. For all strains, corn cobs (CC) was the most appropriate carbon source for the production of bioactive small molecules. Among the biomass extracts, P. citrinopileatus showed the most interesting skin whitening activity $(32.00 \pm 0.92 \%)$ followed by G. resinaceum $(27.25 \pm 0.96 \%)$ and by A. biennis $(25.20 \pm$ $1.85 \%$ ) (Fig. 1). The use of OOMW as carbon source slightly descreased the anti-tyrosinase activity of the EtOAc extracts from the biomasses of G. resinaceum and of A. biennis, while for P. citrinopileatus a complete loss of bioactivity was observed (Fig. 1). Similarly, OOMW leads to the reduction of the anti-aging potential of supernatant extracts. The use of CC was also proved as the most appropriate for maximizing the anti-tyrosinase potential of culture supernatants (Fig. 1). The DPPH scavenging potential of the genarated extracts has significantly decreased when the OOMW was used as carbon source (Fig. 2). However, in contrast to the anti-tyrosinase potential of the EtOAc-LL extracts, supenatants did not show any significant scavanging effect against the DPPH radical at $200 \mu \mathrm{g} \mathrm{mL}^{-1}$ (Fig. 2). These data suggest CC as the more appropriate carbon source for the production of antioxidant and anti-tyrosinase compounds from the aforementioned biomasses. 


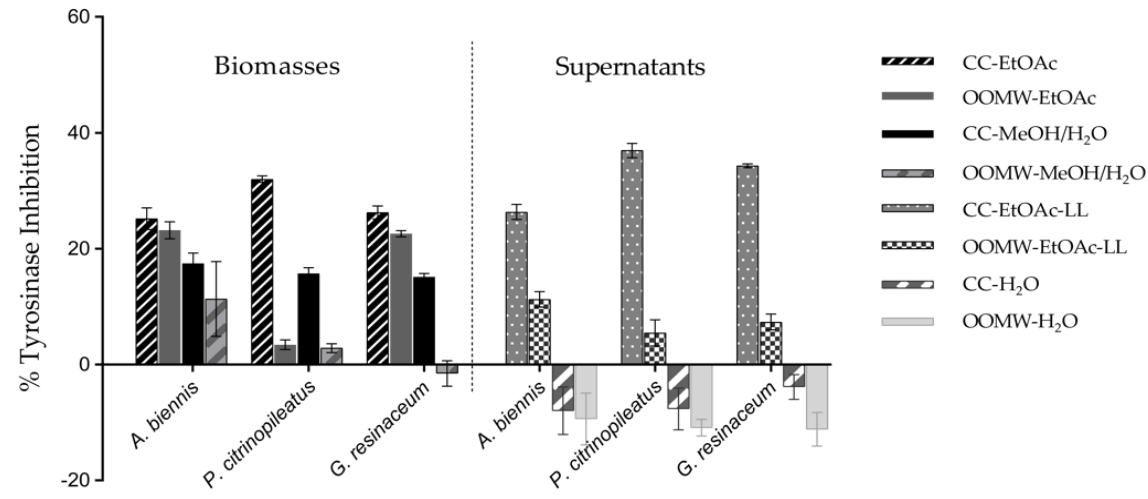

Figure 1. Anti-tyrosinase evaluation of fungal cultures (300 $\left.\mathrm{g} \mathrm{mL}^{-1}\right)$. Positive control: Kojic Acid (2 $\mathrm{ug} / \mathrm{ml}), \%$ inhibition $=44.40 \pm 1.37$; Experiments were performed in triplicate; Error bars indicate \pm standard errors.

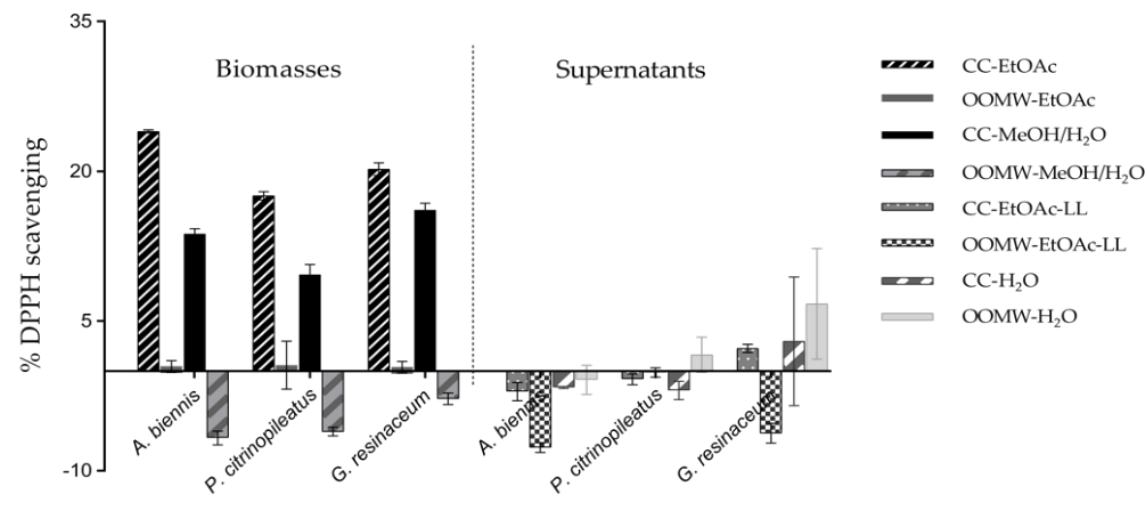

Figure 2. Anti-oxidant evaluation of fungal cultures $\left(200 \mu \mathrm{g} \mathrm{mL} \mathrm{m}^{-1}\right)$. Positive control: Gallic Acid (5 $\mathrm{ug} / \mathrm{ml}), \%$ inhibition $=46.33 \pm 1.51$. Experiments were performed in triplicate; Error bars indicate \pm standard errors.

The first step towards exploring the enzymatic variability of the studied strains in regard to lignocellulose degradation was to assemble the predicted CAZymes of the available genome sequences. The annotation was performed for the publicly available genome sequences of $\mathrm{P}$. citrinopileatus and A. biennis, as shown in Table 1.

Table 1. Predicted CaZymes in the A. biennis and P. citrinopileatus genome.

\begin{tabular}{lcccc}
\hline & Specific activity & CaZy modules & \multicolumn{2}{c}{$\begin{array}{c}\text { No of predicted } \\
\text { sequences }\end{array}$} \\
\hline \multirow{2}{*}{ Cellulases } & & $\begin{array}{c}\text { A. } \\
\text { biennis }\end{array}$ & $\begin{array}{c}\text { P. } \\
\text { citrinopileatus }\end{array}$ \\
& Endoglucanases & GH 5, 7, 12, 45 & 14 & 17 \\
& Cellobiohydrolases & GH 6, 7 & 4 & 10 \\
Xylanases & $\beta$ - glucosidases & GH 1, 3 & 2 & 5 \\
& Endoxylanases & GH 10, 11 & 6 & 15 \\
& $\beta$ - xylosidases & GH 3, 43 & 4 & 7 \\
& Laccases & AA 1 & 8 & 16 \\
& Peroxidases & AA 2 & 12 & 11 \\
& Lytic polysaccharide & AA 9, 10, 11, 13, & 12 & 28 \\
\hline
\end{tabular}


Both genomes include all the necessary genes for lignocellulose breakdown, in multiple copies. However, the existence of the required genes does not necessarily lead to secretion of the respective proteins. Therefore, both strains together with G. resinaceum, which is a strain with unknown genome sequence, were grown in lignocellulosic materials, and the profile of enzyme activities was studied.

OOMW is the liquid by-product of olive oil production by three-phase decanters, popular in Mediterranean countries. White-rot basidiomycetes have been extensively used for the biological treatment of such effluents, due to their potent oxidative enzymes. All of the studied strains achieved complete breakdown of OOMW phenols (Fig. 3).
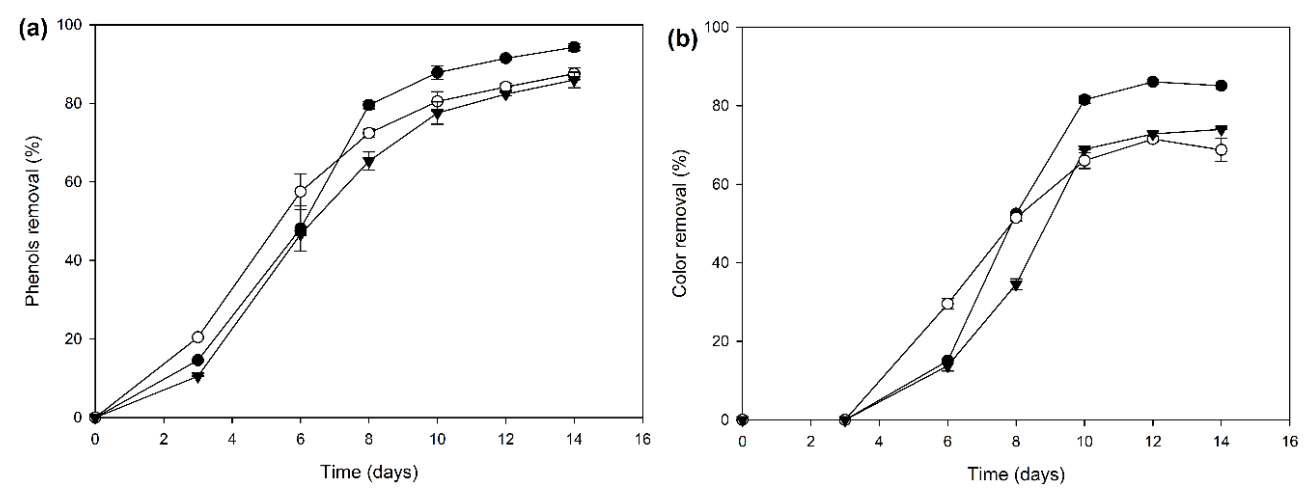

Figure 3. OOMW degradation during growth of P. citrinopileatus (black circles), A. biennis (white circles) and G. resinaceum (black inverted triangles), on OOMW. (a) Removal of phenols, (b) decolorization.

The time profile of laccase production is shown in Fig. 4a. Surprisingly, P. citrinopileatus was found to be the least potent laccase producer. G. resinaceum showed the highest activity $(2.1 \pm 0.04 \mathrm{U}$ $\mathrm{mL}^{-1}$ ).

The enzyme production was also tested during growth on CC, an untreated lignocellulosic substrate. Endocellulase, endoxylanase, and laccase activities were monitored during the course of the culture. The results for laccase activity are shown in Fig. $4 \mathrm{~b}$. Laccase activity for all strains was found to be significantly lower than when grown on OOMW, which is expected due to the low lignin content of the substrate. Again, G. resinaceum was found to be the most potent laccase producer. Surprisingly, endocellulase and endoxylanase activities were not detected at any time point during the course of the fungal cultures. This result might be partly explained by the physiology of whiterot basidiomycetes, most of which are preferential lignin degraders [1].
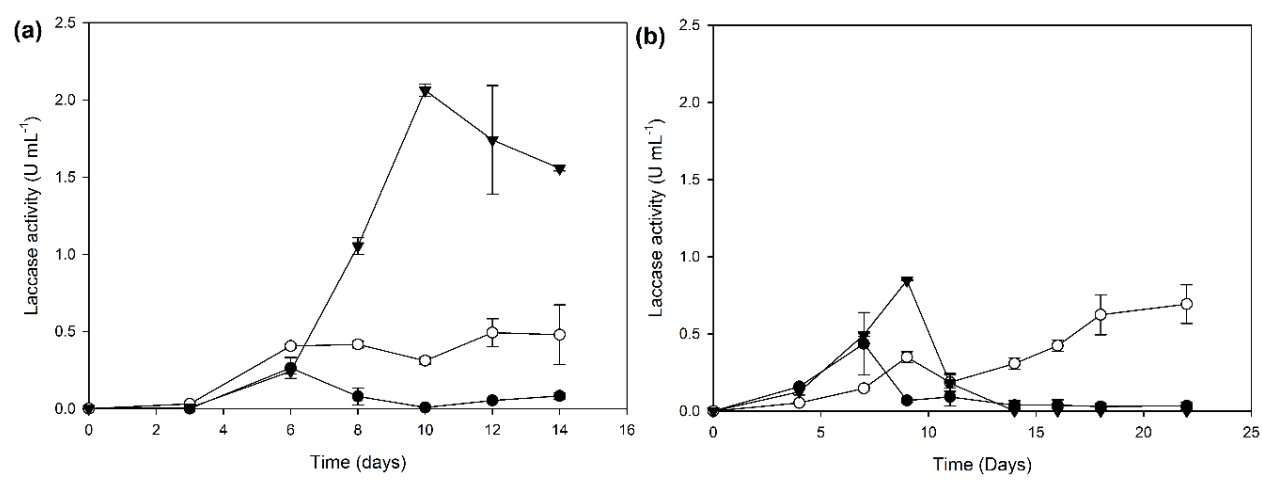

Figure 4. Laccase activity during the growth of P. citrinopileatus (black circles), A. biennis (white circles) and G. resinaceum (black inverted triangles), on OOMW (a) and corn cobs (b).

Despite the absence of detectable endocellulase or endoxylanase activity, soluble reducing sugars were detected in the supernatants, up to $0.66 \mathrm{mg} \mathrm{mL}^{-1} \pm 0.004$ for P. citrinopileatus, $0.59 \pm 0.04$ $\mathrm{mg} \mathrm{mL}^{-1}$ for A. biennis and $0.41 \pm 0.01 \mathrm{mg} \mathrm{mL}^{-1}$ for G. resinaceum, indicating the presence of at least 
some activity on polysaccharides. All the above data, together with the presence of several LPMO geneo in the genomes of $P$. citrinopileatus and A. biennis, support the existence of potent LPMO activity. In order to detect LPMO activity, the three fungal strains were grown in microcrystalline cellulose as sole carbon source. After 15 days of growth, the supernatants were analyzed for LPMO activity (Fig. 5). LPMO activity was detected in the supernatants of all fungal strains [13]. Our results confirm the expression of LPMOs from the studied strains for cellulose degradation, however, the exact role and significance of LPMO activity during fungal growth in natural complex substrates is still unknown.

(a)

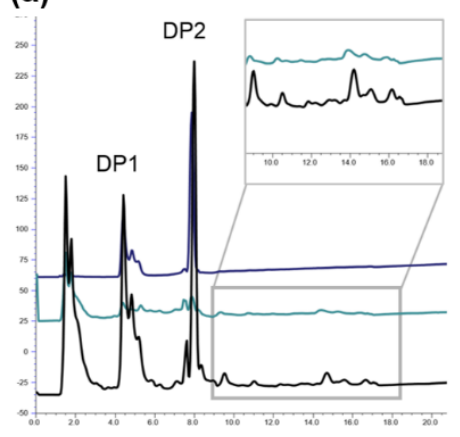

(b)

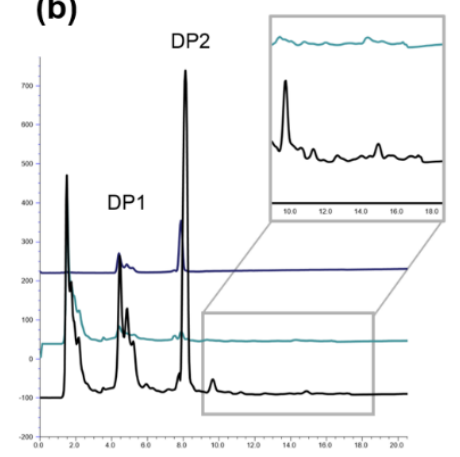

(c)

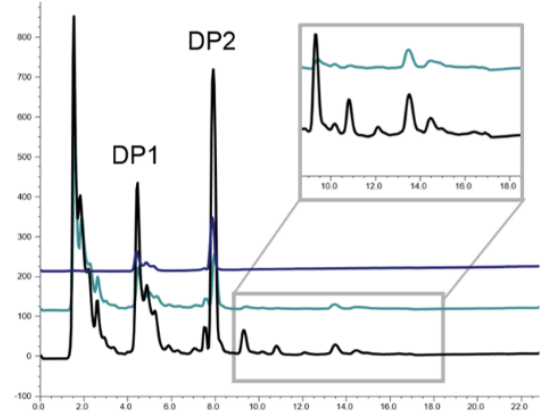

Figure 5. HPAEC-PAD chromatograms for the detection of LPMO activity. (a) P. citrinopileatus, (b) G. resinaceum, (c) A. biennis. Dark blue line: glucose and cellobiose controls, light blue line: reaction control with deactivated enzyme, black line: reaction of crude enzyme with PASC substrate.

\section{Conclusions}

In the present work, a screening study was performed for three white-rot basidiomycete strains, P. citrinopileatus, A. biennis and G. resinaceum in two agroindustrial byproducts: OOMW and corn cobs. P. citrinopileatus showed the highest skin whitening activity, while A. biennis the highest DPPH scavenging potential. Corn cobs was the most appropriate carbon source for the production of bioactive molecules with anti-aging activity. All strains showed significant degradative potential, by removing OOMW phenols, and by the production of laccase activity. Also, they were found to be preferential lignin degraders, similarly to most white-rot fungi. Automated annotation tools revealed that $P$. citrinopileatus and $A$. biennis contain multiple copies of all the necessary genes for complete lignocellulose breakdown. Overall, all strains show promising antiaging proprieties and significant potential for biodegradation applications, but also for the discovery of novel enzyme activities.

Author Contributions: Conceptualization, E.T; methodology, A.Z. \& N.T.; investigation, A.Z. \& N.T.; writing, A.Z. \& N.T..; visualization, A.Z.; supervision, E.T.

Funding: This research is funded in the context of the project "Biotechnological exploitation of wild strains of Greek basidiomycetes for the production of bioactive small molecules and macromolecules with applications in cosmetic and biocatalysis industries - BIO2" (MIS 5049430) under the call for proposals "Researchers' support with an emphasis on young researchers- 2nd Cycle". The project is co-financed by Greece and the European Union (European Social Fund- ESF) by the Operational Programme Human Resources Development, Education and Lifelong Learning 2014-2020.

Acknowledgments: Prof. George Zervakis, Agricultural University of Athens, Department of Crop Science, is gratefully acknowledged for the donation of Pleurotus citrinopileatus LGAM 28684, Abortiporus biennis LGAM 436 and Ganoderma resinaceum LGAM 334 strains.

Conflicts of Interest: The authors declare no conflict of interest. The funders had no role in the design of the study; in the collection, analyses, or interpretation of data; in the writing of the manuscript, or in the decision to publish the results.

\section{References}


1. Schilling, J.S.; Kaffenberger, J.T.; Held, B.W.; Ortiz, R.; Blanchette, R.A. Using Wood Rot Phenotypes to Illuminate the "Gray" Among Decomposer Fungi. Front Microbiol 2020, 11

2. Koutrotsios, G.; Larou, E.; Mountzouris, K.C.; Zervakis, G.I. Detoxification of Olive Mill Wastewater and Bioconversion of Olive Crop Residues into High-Value-Added Biomass by the Choice Edible Mushroom Hericium erinaceus. Appl Biochem Biotechnol 2016 180, 195-209

3. Georgousaki, K.; Tsafantakis, N.; Gumeni, S.; Gonzalez, I.; Mackenzie, T.A.; Reyes, F.; Lambert, C.; Trougakos, I.P.; Genilloud, O.; Fokialakis, N. Screening for tyrosinase inhibitors from actinomycetes; identification of trichostatin derivatives from Streptomyces sp. CA-129531 and scale up production in bioreactor. Bioorganic Med. Chem. Lett. 2020, 30, 126952

4. Zolghadri, S.; Bahrami, A.; Hassan Khan, M.T.; Munoz-Munoz, J.; Garcia-Molina, F.; Garcia-Canovas, F.; Saboury, A.A. A comprehensive review on tyrosinase inhibitors. J. Enzyme Inhib. Med. Chem. 2019, 34, 279309

5. Ntougias, S.; Gaitis, F.; Katsaris, P.; Skoulika, S.; Iliopoulos, N.; Zervakis, G.I. The effects of olives harvest period and production year on olive mill wastewater properties: evaluation of Pleurotus strains as bioindicators of the effluent's toxicity. Chemosphere, 2013, 92, 399-405

6. Zerva, A.; Zervakis, G.I.; Christakopoulos, P.; Topakas, E. Degradation of olive mill wastewater by the induced extracellular ligninolytic enzymes of two wood-rot fungi. J environ manage 2017, 203, 791-798

7. Zerva, A.; Savvides, A.L.; Katsifas, E.A.; Karagouni, A.D.; Hatzinikolaou, D.G. Evaluation of Paecilomyces variotii potential in bioethanol production from lignocellulose through consolidated bioprocessing. Bioresour technol 2014, 162, 294-299

8. Miller, GL. Use of dinitrosalicylic acid reagent for determination of reducing sugar. Anal Chem 1959, 31(3), 426-8.

9. Zerva, A.; Pentari, C.; Grisel, S.; Berrin, J.-G.; Topakas, E. A new synergistic relationship between xylanactive LPMO and xylobiohydrolase to tackle recalcitrant xylan. Biotechnol Biofuels 2020, 13, 142

10. Zhang, H.; Yohe, T.; Huang, L.; Entwistle, S.; Wu, P.; Yang, Z.; Busk, P.; Xu, Y.; Yin, Y. dbCAN2: a meta server for automated carbohydrate-active enzyme annotation, Nucleic Acids Res, 2018, 46, W95-W101

11. Lee, S.E.; Hwang, H.J.; Ha, J.S.; Jeong, H.S.; Kim, J.H. Screening of medicinal plant extracts for antioxidant activity. Life Sci. 2003, 73, 167-179

12. Chaita, E.; Lambrinidis, G.; Cheimonidi, C.; Agalou, A.; Beis, D.; Trougakos, I.; Mikros, E.; Skaltsounis, A.L.; Aligiannis, N. Anti-Melanogenic Properties of Greek Plants. A Novel Depigmenting Agent from Morus alba Wood. Molecules 2017, 22, 514

13. Villares, A.; Moreau, C.; Bennati-Granier, C.; Garajova, S.; Foucat, L.; Falourd, X.; Saake, B.; Berrin, J.G.; Cathala, B. Lytic polysaccharide monooxygenases disrupt the cellulose fibers structure. Sci Rep 2017, 7, 40262 .

Publisher's Note: MDPI stays neutral with regard to jurisdictional claims in published maps and institutional affiliations.

(C) 2020 by the authors; licensee MDPI, Basel, Switzerland. This article is an open access article distributed under the terms and conditions of the Creative Commons Attribution (CC-BY) license (http://creativecommons.org/licenses/by/4.0/). 